\title{
Comparación de los baremos del CUMANIN y CUMANES: una experiencia psicométrica ${ }^{1}$
}

\author{
Julian David Chinome Torres ${ }^{2}$, Lucía Carlota Rodríguez Barreto ${ }^{3}$ \\ Universidad Pedagógica y Tecnológica de Colombia-Colombia
}

\begin{abstract}
Se hizo una valoración neuropsicológica con los cuestionarios CUMANIN y CUMANES usando los baremos colombianos, haciendo una comparación con los baremos originales. El estudio es descriptivo-no experimental, en total, participaron 400 niños de colegios públicos y privados de 6 municipios del departamento de Boyacá-Colombia, Los participantes tenían edades entre los 3 y 11 años $(M=6,69 ; \mathrm{DE}=2,32$ años). Se realizó un análisis de desempeño a partir de frecuencias en cada una de las subescalas que contaban con baremo, se hizo un análisis comparativo con la prueba t-student. Se identificaron diferencias estadísticas en el uso de los baremos en el CUMANIN y CUMANES. Se encontraron diferencias en lenguaje articulatorio en el CUMANIN en las variables sexo y tipo de institución. En el CUMANES se encontraron diferencias en el tipo de institución. Se sugiere hacer uso de los baremos de acuerdo a lo sugerido por la APA, se recomienda tener precauciones en el uso de pruebas no baremadas. El uso de los baremos permitiría la elaboración de algunas políticas públicas en la infancia a partir de una aproximación diagnóstica válida.

Palabras clave: evaluación neuropsicológica, desarrollo infantil, psicometría.
\end{abstract}

\section{Comparison of the CUMANIN and CUMANES Scales: A psychometric experience}

Se hizo una valoración neuropsicológica con los cuestionarios CUMANIN y CUMANES usando los baremos colombianos, haciendo una comparación con los baremos originales. El estudio es descriptivo-no experimental, en total, participaron 400 niños de colegios públicos y privados de 6 municipios del departamento de Boyacá-Colombia, Los participantes tenían edades entre los 3 y 11 años $(M=6,69 ; \mathrm{DE}=2,632$ años). Se realizó un análisis de desempeño a partir de frecuencias en cada una de las subescalas que contaban con baremo, se hizo un análisis comparativo con la prueba t-student. Se identificaron diferencias estadísticas en el uso de los baremos en el CUMANIN y CUMANES. Se encontraron diferencias en lenguaje articulatorio en el CUMANIN en las variables sexo y tipo de institución. En el CUMANES se encontraron diferencias en el tipo de institución. Se sugiere hacer uso

1 Este artículo es parte de los productos de un proyecto de investigación aprobado por la Vicerrectoría de Investigación y Extensión Universitaria de la Universidad Pedagógica y Tecnológica de Colombia. Código SGI- 2337

2 Licenciado en Psicología. Joven talento investigador, Universidad Pedagógica y Tecnológica de Colombia. Dirección postal: Sede Central Tunja-Boyacá-Colombia Avenida Central del Norte 39-115. Contacto: juliandavid.chinome@uptc.edu.co https://orcid. org/0000-0003-3467-7055

3 Doctora en Neurociencias. Docente asociada Universidad Pedagógica y Tecnológica de Colombia. Dirección postal: Sede Central Tunja-Boyacá-Colombia Avenida Central del Norte 39-115 Contacto: lucia.rodriguezb@uptc.edu.co https://orcid.org/0000-0003-1306-6909 
de los baremos de acuerdo a lo sugerido por la APA, se recomienda tener precauciones en el uso de pruebas no baremadas. El uso de los baremos permitiría la elaboración de algunas políticas públicas en la infancia a partir de una aproximación diagnóstica válida. Keywords: Neuropsychological assessment; child development, Psychometry.

\section{Comparaçáo das escalas CUMANIN e CUMANES: uma experiência psicométrica}

Foi realizada uma avaliação neuropsicológica com os questionários CUMANIN e CUMANES, utilizando as escalas colombianas, fazendo uma comparação com as escalas originais. Participaram do estudo descritivo-não-experimental, totalizando 400 crianças de escolas públicas e privadas de 6 municípios do departamento de Boyacá-Colômbia, com idade entre 3 e 11 anos $(M=6,69$; $\mathrm{DP}=2.632$ anos $)$. Uma análise de desempenho foi realizada com base nas frequências em cada uma das subescalas que possuíam escala; uma análise comparativa foi realizada com o teste t-student. Foram identificadas diferenças estatísticas no uso das escalas no CUMANIN e CUMANES. Foram encontradas diferenças na linguagem articulatória na CUMANIN nas variáveis sexo e tipo de instituição. Em CUMANES foram encontradas diferenças no tipo de instituição. Recomenda-se o uso das balanças, conforme sugerido pela APA, é recomendável tomar precauções no uso de testes não relacionados à balança. $\mathrm{O}$ uso de escalas permitirá a elaboração de algumas políticas públicas na infância a partir de uma abordagem diagnóstica válida.

Palavras-chave: avaliação neuropsicológica; desenvolvimento infantil, psicometria.

\section{Comparaison des échelles CUMANIN et CUMANES: une expérience psychométrique} Une évaluation neuropsychologique a été réalisée avec les questionnaires CUMANIN et CUMANES en utilisant les échelles colombiennes, en faisant une comparaison avec les échelles originales. L'étude est descriptive et non expérimentale. Au total, 400 enfants d'écoles publiques et privées de 6 municipalités du département de Boyacá-Colombia ont participé, âgés de 3 à 11 ans $(M=6,69 ; S D=2632$ années). Une analyse de performance a été réalisée sur la base des fréquences de chacune des sous-échelles à l'échelle, une analyse comparative a été réalisée avec le test t-student. Des différences statistiques dans l'utilisation des échelles dans CUMANIN et CUMANES ont été identifiées. Des différences de langage articulatoire ont été observées dans CUMANIN dans les variables sexe et type d'établissement. En CUMANES, des différences ont été observées dans le type d'établissement. Il est recommandé d'utiliser les balances suggérées par l'APA. Il est également recommandé de prendre des précautions lors de l'utilisation de tests sans balances. L'utilisation d'échelles permettra d'élaborer certaines politiques publiques dans l'enfance à partir d'une approche diagnostique valide.

Mots-clés: Évaluation neuropsychologique; développement de l'enfant, psychométrie. 
A pesar de los esfuerzos gubernamentales e institucionales a nivel mundial, existe debilidades en la creación de programas para la atención en primera infancia, que se centra en países con ingresos bajos. Se propone un consenso en la delimitación del problema y sus posibles alternativas, con miras a financiación de tipo político y creación de instituciones efectivas que logren desarrollar objetivos comunes e intersectoriales que atiendan a esta población (Shawar y Shiffman, 2017).

Aproximadamente 250 millones (43\%) de niños menores de 5 años residentes en países con ingresos medios y bajos, no alcanzan un desarrollo madurativo óptimo (Walker et al., 2007, 2011a). Los avances en neurociencias vinculan las adversidades tempranas con dificultades en el desarrollo cerebral, la primera infancia y la calidad de vida en general, se hace necesario un abordaje multisectorial e interdisciplinar incluyendo estrategias psicosociales que logren garantizar el adecuado desarrollo intelectual, creativo y social para un desarrollo deseable (Black et al., 2016).

Se estima que los niños que han tenido factores de riesgo, un desarrollo incompleto y no acceden a dichos programas, en su adultez aportarán muy poco al PIB de su país, ganarían una cuarta parte de los ingresos promedio de los adultos en su país y recibirían más ayudas por parte de sus gobiernos (Richter et al., 2017). En algunas oportunidades, los niños experimentan la presencia de trastornos psiquiátricos y mayor probabilidad de sufrir comorbilidades, dificultades en el desempeño escolar, fracaso y abandono escolar y dificultades de tipo socioeconómico (Ackerman y Brown, 2006; Heckman y Masterov, 2007; Nelson et al., 2009).

En estudios de Cermakova et al. (2018) se sugiere que los adultos que experimentaron dificultades socioeconómicas en la infancia tenían un peor desempeño cognitivo en la adultez, aumentando consigo sus dificultades socioeconómicas a lo largo de su vida. La posición socioe- 
conómica desprotegida durante la infancia también se relacionó con un envejecimiento cognitivo mayor (Fritze, Doblhammer y van den Berg, 2014). Por su parte en un estudio longitudinal con participantes entre los 5 y 25 años se encontró relación entre el estatus socioeconómico y densidad de estructuras cortico-subcorticales a través de imágenes de resonancia magnética estructural (sMRI) (McDermott et al., 2018). A partir de estas desventajas socioeconómicas existe una mayor probabilidad de no contar con acceso a servicios de salud integral, una valoración neuropsicológica infantil completa y un seguimiento adecuado.

Autores como Lenroot y Giedd (2006) y Rees, Booth y Jones (2016) explican que en la edad de los 0 hacia los 5 y 6 años se evidencian unos picos de plasticidad cerebral, ya que en este tiempo existe una ganancia de peso cerebral y cambios a nivel de materia gris y blanca. La implementación de programas en estas edades mitigarían algunos déficits asociados a las experiencias adversas tempranas, o fortalecerían las funciones cognitivas normales (Shonkoff, 2011; Bryck y Fisher, 2012). Durante los primeros años de vida especialmente hasta los 3 años, se encuentran factores determinantes relacionados con el desarrollo físico, psicosocial, emocional y cognitivo de los niños los cuales perdurarán por el resto de sus vidas, permitiendo la neurogénesis de millones de neuronas y la sinaptogénesis de millones de conexiones neurales (Pérez-Escamilla et al.,2017).

Relacionado con este nivel de desarrollo madurativo, se asocia el término Madurez Neuropsicológica ( $\mathrm{MN}$ ), entendida como el nivel de organización y desarrollo cerebral que corresponde a una edad cronológica específica (Moreno-Gómez y Cejudo, 2018; Portellano Pérez et al., 2000).

La MN permite evidenciar una organización funcional a través de los procesos cognitivos y conductuales que pueden ser observables y evaluados a través de test de tipo psicométrico no invasivos y sensibles a este constructo (Chinome Torres et al., 2017; Parra-Pulido, RodríguezBarreto y Chinome-Torres, 2016). 
Algunas de las dificultades experimentadas a lo largo del desarrollo infantil que se han relacionado con la presencia de inmadurez neuropsicológica son el peso al nacer (Parra Pulido, Rodríguez Barreto y Chinome Torres, 2015; Savanur, Purandare y Ghugre, 2015); la pobreza y el estatus socioeconómico y familiar (Cermakova et al., 2018; Johnson, Riis y Noble, 2016; McDermott et al., 2018) el grado de escolaridad de los padres (Noble et al., 2015; Sun, McHale y Updegraff, 2017).

Otras relacionadas con procesos de tipo fisiológico son el historial de desnutrición parental (Waber et al., 2018) funciones metabólicas cerebrales, desnutrición y procesos infecciosos (John, Black y Nelson, 2017; Peng et al., 2015; Schoenmaker et al., 2015).

La valoración neuropsicológica es una herramienta importante para evidenciar el estado de algunas capacidades cognitivas como la atención, la memoria, las funciones ejecutivas relacionado con un posible daño cerebral o simplemente analizar estos constructos en términos de normalidad (Solís-Rodríguez et al., 2018). En países en vía de desarrollo los test neuropsicológicos escasean, además, en su mayoría no tienen un alcance deseable en población infantil, diversos test son para adultos y pacientes hospitalizados (Nell, 2000).

Para Arango-Lasprilla y Rivera (2015) en Colombia no existe una buena cantidad de pruebas neuropsicológicas, muchas no cuentan con los estudios psicométricos para cada población en particular, generando un sesgo en la aproximación diagnóstica. La baremación de pruebas neuropsicológicas en el país es una deuda actualmente y el uso de los existentes es escaso (Ardila y Ostrosky, 2012).

Es deseable contar con test diagnósticos adecuados, evaluar la madurez neuropsicológica con los estudios psicométricos en la población objetivo permitirá dar un abordaje ideal en cada caso específico, ya que la $\mathrm{MN}$ es un factor importante para disminuir dificultades a largo plazo, es ideal en la detección oportuna de trastornos específicos de aprendizaje (TEA) que se ha relacionado con el fracaso y abandono escolar, lo cual trae consigo problemáticas de tipo social. 
Según recomendaciones de Martín Casari y Oros Cabrini (2013) se debe tener precaución en la interpretación que se hacen de los resultados de las pruebas psicométricas aplicadas que no cuentan con una estandarización, sugieren también en hacer la baremación de pruebas neuropsicológicas, ya que esto permitirá hacer una aproximación a un diagnóstico, debido a las influencias socioculturales.

El objetivo principal en el presente estudio fue aplicar los baremos obtenidos en estudios previos en población Colombiana de los instrumentos CUMANES y CUMANIN, Cuestionario de Madurez Neuropsicológica Escolar (Portellano et al., 2012) y Cuestionario de Madurez Neuropsicológica Infantil (Portellano Pérez et al., 2000) en una muestra de niños y niñas de 3 a 11 años, residentes en el departamento de Boyacá.

\section{Método}

\section{Participantes}

La selección de la muestra fue de tipo intencional. Estuvo conformada por 400 niños (as) con edades comprendidas entre los 3 y 11 años, la media de la edad fue de 6.690 años, con una desviación típica de (2.63 años) ( $M E=.69$ años; $D E=2.63$ años). En total 199 participantes eran mujeres (49.75\%) y 201 hombres (50.25\%) (Tabla 1 ), eran estudiantes de instituciones públicas y privadas, de 6 municipios del departamento de Boyacá de las provincias Centro y Tundama. Para esta investigación los criterios de inclusión fueron: (a) tener edades comprendidas entre los 3 y 11 años al momento de la ejecución de la investigación (b) saber leer y escribir únicamente para participantes del CUMANES y para algunas subescalas del CUMANIN que así lo requiriera (c) tener autorización por parte de sus padres y/o representante legal para la participación de este estudio con la firma del consentimiento informado y (d) que el participante no tuviera alguna limitación motriz o sensorial que impidiera realizar la prueba. 


\section{Consideraciones éticas}

Esta investigación tiene el aval del comité de ética de la Vicerrectoría de Investigaciones y Extensión (VIE) de la Universidad Pedagógica y Tecnológica de Colombia. El consentimiento informado incluye los lineamientos de la Ley 1090 de 2006 en el literal D, del artículo 25, los padres de familia o tutores legales lo firmaron en la reunión de socialización del proyecto, respondiendo previamente a las inquietudes.

\section{Medición}

El CUMANIN, según Colmenero y Robles Bello (2012) es uno de los test neuropsicológicos más utilizados en la actualidad ya que permite identificar posibles trastornos de tipo madurativo relacionados con dificultades de tipo cognitivo cuya base presumible es de tipo neuroanatómico. Este es un test utilizado en población pediátrica con o sin alteraciones (Martínn-Casas et al., 2015, 2016; Millana-Cuevas., Portellano, 2007), se han realizado diversas adaptaciones de este cuestionario incluyendo las de Guerrero-Leiva (2006) y Urzúa et al. (2010).

El CUMANIN está conformado por 83 ítems distribuidos en 8 escalas principales, y 44 ítems que constituyen 5 subescalas adicionales, entre estas, la subescala de lateralidad que mide el uso preferente de mano ojo y pie, para un total de 13 subescalas, la calificación por ítem es dicotómico, con acierto (1) o error (0), va dirigido a nińos con edades comprendidas entre los 36 y 78 meses, la ejecución de la prueba por cada subescala, es interpretada de acuerdo a los centiles, según los autores las puntuaciones se distribuyen así: baja si la puntuación está entre los centiles 5-39, un valor medio entre los centiles 40-60 y un desempeño alto o superior entre los centiles 61-99 (ver tabla 3), en cuanto al coeficiente de desarrollo la interpretación se realiza a partir de la media 100 con una desviación típica de 10 puntos.

Se usaron las adaptaciones de equivalencia semántica y cultural hechas por (Ávila, 2012)aporta a este propósito; en Perú, se realizó una adaptación con buenos resultados; el objetivo de este estudio fue realizar la adaptación para Colombia. Para ello, se realizó aplicación piloto, 
validación por jueces (coeficiente kappa en una población colombiana de 303 niños. El índice alfa de Cronbach de la prueba original oscila entre .71 y 0.92 , esto indica que es aceptable.

El CUMANES (Portellano, Mateos y Martínez-Arias, 2012) es un test dirigido a nińos entre los 7 y 11 años de edad y es la continuidad del CUMANIN, ha sido calificado por Zamora Crespo y García-Navarro (2014) como uno de los cuestionarios desarrollados específicamente para la población infantil.

En la actualidad es uno de las más utilizadas en la población pediátrica, y población hispano-hablante (Benítez et al., 2015).

Su aplicación es de manera individual, su finalidad es evaluar de forma global el desarrollo neuropsicológico en el ámbito clínico o educativo. Se obtiene una puntuación directa a partir de la suma de las respuestas correctas con modalidad dicotómicas o politómicas. Cuenta con baremos originales para niños de España, descritos en modo de decatipos para cada una de las pruebas y percentiles de la puntuación total en cada grupo de edad.

La prueba tiene 12 subescalas y 6 áreas, dentro del Lenguaje se encuentran las subescalas de (comprensión audio-verbal, comprensión de imágenes, fluidez fonológica, fluidez semántica, leximetría [comprensión y velocidad lectora] y escritura audiognósica), las otras escalas son visopercepción, funcionamiento ejecutivo (tiempo y errores), memoria (verbal y visual) y ritmo. A partir del desempeño en cada escala se obtiene una puntuación global que resume el nivel de madurez neuropsicológica en este caso el Índice de Desarrollo Neuropsicológico (IDN), cuenta también con escala de lateralidad, que en este caso no es necesaria para la obtención del IDN.

Para la calificación se utilizaron los baremos diseñados por ParraPulido y Rodríguez-Barreto (2016) en una muestra colombiano, cuenta con la baremación por algunas subescalas del cuestionario original, estas son: psicomotricidad, lenguaje articulatorio, memoria icónica, ritmo, lenguaje comprensivo, estructuración espacial y visopercepción. Dicho manuscrito investigativo se encuentra actualmente en proceso de publicación. A su vez se utilizaron los baremos del CUMANES diseñados 
por Chinome-Torres, Rodríguez-Barreto y Pineda-Roa (2018) también en proceso de publicación, estos baremos constituyen un cambio en la valoración total del desempeño expresado en el IDN, pues se adaptaron las puntuaciones típicas y los percentiles, dando un valor aproximado y válido en la población utilizada.

\section{Procedimiento}

Se realizó una investigación de tipo descriptivo y cuantitativo (Hernandez Sampieri, Fernandez Collado y Baptista Lucio, 2010). El diseño de la investigación fue un estudio no experimental ya que se desea describir los resultados de la valoración neuropsicológica utilizando los baremos para una población colombiano.

Se realizó el contacto pertinente con las instituciones educativas privadas y públicas de los municipios seleccionados, con el fin de presentar la propuesta de investigación, en la socialización se acordaron los tiempos de la aplicación y la selección de espacios adecuados para cada aplicación teniendo en cuenta los factores medioambientales.

Se estableció contacto con los padres de los participantes a quienes se les socializó el procedimiento y dinámicas del proyecto, también se informó los objetivos, los derechos como participantes, deberes como investigadores y las normas éticas que rigen la investigación firmando el consentimiento informado; se llevó a cabo la aplicación del cuestionario de forma individual, en un espacio y tiempo apropiado en donde solo se encontraba el investigador y el participante, de acuerdo con las recomendaciones de los autores.

Por último, se entregaron los informes a cada uno de los padres de familia, previa citación en cada una de las instituciones educativas, el informe incluía una serie de recomendaciones y estrategias en casa para promover el desarrollo neurocognitivo del niño.

\section{Análisis de datos}

Para el análisis del CUMANIN se realizó un análisis descriptivo, consistió en calcular las medias de cada una de las subescalas que habían sido baremadas, en seguida se compararon las puntuaciones centiles de 
los baremos originales con los centiles de los baremos obtenidos en la muestra colombiana, esto a partir de una prueba $t$ de student para muestras independientes, detectando así las posibles diferencias basadas en este desempeño, a través del paquete estadístico SPSS ${ }^{\odot}$.

En cuanto al CUMANES, se realizó un análisis de desempeño basado en las frecuencias a partir de los decatipos baremados, se hizo también, un análisis comparativo entre las puntuaciones del IDN y el percentil de los baremos originales y la versión baremada para Colombia. Por último, se realizó un análisis comparativo entre las puntuaciones en las subescalas baremadas en población colombiano del CUMANIN y del CUMANES entre las variables sexo y tipo de institución en cada cuestionario a partir del estadístico t-student para muestras independientes a través del paquete estadístico SPSS.

\section{Resultados}

\section{Tabla 1}

Distribución de la muestra en función de la edad y el sexo.

\begin{tabular}{ccccccc}
\hline \multirow{2}{*}{ Edad años } & \multicolumn{3}{c}{ Sexo } & \multicolumn{3}{c}{ Total } \\
\cline { 2 - 5 } & \multicolumn{2}{c}{ Masculino } & \multicolumn{2}{c}{ Femenino } & & \multicolumn{3}{c}{ n } \\
\cline { 2 - 5 } 3 & $\mathrm{n}$ & $\%$ & $\mathrm{n}$ & $\%$ & 54 & 13.5 \\
4 & 30 & 7.5 & 24 & 6 & 54 & 13.5 \\
5 & 28 & 7 & 26 & 6.5 & 54 & 13.5 \\
6 & 21 & 5.25 & 17 & 4.25 & 38 & 9.5 \\
7 & 20 & 5 & 20 & 5 & 40 & 10 \\
8 & 20 & 5 & 20 & 5 & 40 & 10 \\
9 & 20 & 5 & 20 & 5 & 40 & 10 \\
10 & 20 & 5 & 20 & 5 & 40 & 10 \\
11 & 20 & 5 & 20 & 5 & 40 & 10 \\
Total & 201 & 50.25 & 199 & 49.75 & 400 & 100
\end{tabular}


La tabla 1 muestra una distribución balanceada en la composición de la muestra por edad y sexo. Se observa en la tabla 2 el desempeño de los participantes en las subescalas del CUMANIN, de acuerdo con las normas interpretativas expresadas en el manual descritas por Portellano Pérez et al. (2000) de la siguiente manera: una puntuación centil por debajo de 33 indica un desempeño bajo, entre 34 y 66 una puntuación media y de 67 a 99 una puntuación superior.

\section{Tabla 2}

Desempeño según frecuencias en las subescalas con los baremos originales $y$ baremos colombianos del CUMANIN.

\begin{tabular}{|c|c|c|c|c|c|c|c|c|c|c|c|c|c|c|}
\hline \multicolumn{15}{|c|}{ Escalas } \\
\hline \multirow[t]{2}{*}{$\begin{array}{l}\text { Des- } \\
\text { empe- } \\
\text { ño }\end{array}$} & \multicolumn{2}{|c|}{$\begin{array}{l}\text { Psicomotrici- } \\
\text { dad n (\%) }\end{array}$} & \multicolumn{2}{|c|}{$\begin{array}{l}\text { L. Articula- } \\
\text { torio n }(\%)\end{array}$} & \multicolumn{2}{|c|}{$\begin{array}{l}\text { L. Com- } \\
\text { prensivo n } \\
(\%)\end{array}$} & \multicolumn{2}{|c|}{$\begin{array}{l}\text { Estructura- } \\
\text { ción n espa- } \\
\text { cial n }(\%)\end{array}$} & \multicolumn{2}{|c|}{$\begin{array}{l}\text { Visopercep- } \\
\text { ción n (\%) }\end{array}$} & \multicolumn{2}{|c|}{$\begin{array}{c}\text { Memoria } \\
\text { icónica n } \\
(\%)\end{array}$} & \multicolumn{2}{|c|}{ Ritmo n (\%) } \\
\hline & $\mathrm{O}$ & B & $\mathrm{O}$ & B & $\mathrm{O}$ & B & $\mathrm{O}$ & B & $\mathrm{O}$ & B & $\mathrm{O}$ & B & $\mathrm{O}$ & B \\
\hline Bajo & $\begin{array}{c}31 \\
(15.5)\end{array}$ & $\begin{array}{c}23 \\
(11.5)\end{array}$ & $\begin{array}{c}36 \\
(18)\end{array}$ & $\begin{array}{c}51 \\
(25.5)\end{array}$ & $\begin{array}{c}46 \\
(23)\end{array}$ & $\begin{array}{c}64 \\
(32)\end{array}$ & $\begin{array}{c}35 \\
(17.5)\end{array}$ & $\begin{array}{c}56 \\
(28)\end{array}$ & $\begin{array}{c}30 \\
(15)\end{array}$ & $\begin{array}{c}41 \\
(20.5)\end{array}$ & $\begin{array}{l}10 \\
(5)\end{array}$ & $\begin{array}{c}38 \\
(19)\end{array}$ & $\begin{array}{c}89 \\
(44.5)\end{array}$ & $\begin{array}{c}25 \\
(12.5)\end{array}$ \\
\hline Medio & $\begin{array}{c}87 \\
(43.5)\end{array}$ & $\begin{array}{c}80 \\
(40)\end{array}$ & $\begin{array}{c}94 \\
(47)\end{array}$ & $\begin{array}{c}60 \\
(30)\end{array}$ & $\begin{array}{c}75 \\
(37.5)\end{array}$ & $\begin{array}{c}68 \\
(34)\end{array}$ & $\begin{array}{l}52 \\
(26)\end{array}$ & $\begin{array}{l}56 \\
(28)\end{array}$ & $\begin{array}{c}70 \\
(35)\end{array}$ & $\begin{array}{c}71 \\
(35.5)\end{array}$ & $\begin{array}{l}50 \\
(25)\end{array}$ & $\begin{array}{c}44 \\
(22)\end{array}$ & $\begin{array}{l}52 \\
(26)\end{array}$ & $\begin{array}{c}86 \\
(43)\end{array}$ \\
\hline Alto & $\begin{array}{c}82 \\
(41)\end{array}$ & $\begin{array}{c}97 \\
(48.5)\end{array}$ & $\begin{array}{c}70 \\
(35)\end{array}$ & $\begin{array}{c}89 \\
(44.5)\end{array}$ & $\begin{array}{c}79 \\
(39.5)\end{array}$ & $\begin{array}{c}68 \\
(34)\end{array}$ & $\begin{array}{c}113 \\
(56.5)\end{array}$ & $\begin{array}{c}88 \\
(44)\end{array}$ & $\begin{array}{l}100 \\
(50)\end{array}$ & $\begin{array}{c}88 \\
(44)\end{array}$ & $\begin{array}{l}140 \\
(70)\end{array}$ & $\begin{array}{l}118 \\
(59)\end{array}$ & $\begin{array}{c}59 \\
(29.5)\end{array}$ & $\begin{array}{c}89 \\
(44.5)\end{array}$ \\
\hline Total & 200 & 200 & 200 & 200 & 200 & 200 & 200 & 200 & 200 & 200 & 200 & 200 & 200 & 200 \\
\hline
\end{tabular}

O: Baremo original. B: Baremo colombiano

A nivel descriptivo se identifica un desempeño diferenciado en el CUMANIN de acuerdo con el baremo utilizado, en el caso de lenguaje articulatorio, lenguaje comprensivo, estructuración espacial, visopercepción y memoria icónica, cuando se hace uso de los baremos colombianos existen mayores frecuencias en el desempeño bajo frente al uso de los baremos originales, caso contrario sucede en psicomotricidad y ritmo. Por otro lado, en las subescalas de psicomotricidad, lenguaje articulatorio y ritmo, utilizando el baremo colombiano existen mayores frecuencias en el desempeño alto. Mientras que con el uso del baremo original las 
escalas de lenguaje comprensivo, estructuración espacial, visopercepción y memoria icónica poseen mayores frecuencias en el desempeño alto frente al baremo colombiano. Esto cambia significativamente la manera en cómo se va a interpretar el desempeño de los participantes. Con el uso de los baremos originales la subescala con peor desempeño fue Ritmo con un $44.5 \%$, mientras que con los colombianos fue lenguaje comprensivo con un 32\%. La escala de memoria icónica fue la subescala donde existió un mejor desempeño con el uso de los baremos originales con un $70 \%$ y con el uso de los baremos colombianos con un $59 \%$.

La valoración con el CUMANIN en niños de 3 a 6 años, mostró diferencias respecto al uso de los baremos, en promedio el $19.78 \%$ de los participantes obtuvieron puntuaciones bajas en cualquiera de las subescalas, mientras que con el baremo colombiano este mismo promedio fue de $21.2 \%$, el desempeño promedio con el uso del baremo original fue de $34.22 \%$ y en promedio con el uso del colombiano fue de $33.3 \%$, por último, el promedio de desempeño alto con el uso del baremo espańol fue de $46 \%$ mientras que con el colombiano en promedio fue de $45.5 \%$.

Se observan las puntuaciones directas por cada una de las subpruebas del CUMANIN, también se encuentran las medias y diferencia de medias entre las puntuaciones de los baremos originales y el adaptado a la población colombiana en la Tabla 3.

Se encontraron diferencias significativas entre los baremos utilizados, en algunos casos si se siguen las normas interpretativas señaladas con anterioridad cambiarían su aproximación diagnóstica, en algunas subescalas, situación que se asemeja en la Tabla 2.

El uso del baremo espańol favorece el diagnóstico en algunas edades, es el caso de estructuración espacial y memoria icónica en el grupo de 3 ańos, de estructuración espacial en el grupo de edad de 4 años, en estructuración espacial y memoria icónica en los grupos de edad de 5 y 6 años. A su vez, se encontró que el uso de los baremos colombianos también favorece algunas puntuaciones por encima de los espańoles, es el caso de lenguaje articulatorio en el grupo de edad de 3 ańos, ritmo en los grupos de 4, 5 y 6 ańos. 


\section{Tabla 3}

Estadisticos descriptivos de las pruebas del CUMANIN

\begin{tabular}{|c|c|c|c|c|c|c|c|c|}
\hline Edad & Prueba & Min & Max & M & $\mathrm{DE}$ & $\begin{array}{c}\text { M Centil } \\
\text { original }\end{array}$ & $\begin{array}{l}\text { M Centil } \\
\text { Colombia }\end{array}$ & $p$ \\
\hline \multirow{7}{*}{$\begin{array}{c}3 \\
\text { ańos }\end{array}$} & Psicomotricidad & 3.0 & 10.0 & 6.07 & 1.52 & 63.61 & 61.79 & .689 \\
\hline & Lenguaje articulatorio & .0 & 15.0 & 6.13 & 4.98 & 46.03 & 58.94 & $.011^{*}$ \\
\hline & Lenguaje comprensivo & .0 & 8.0 & 3.59 & 2.00 & 71.31 & 63.83 & .08 \\
\hline & Estructuración espacial & 4.0 & 13.0 & 7.11 & 1.89 & 72.31 & 61.96 & $.02^{*}$ \\
\hline & Visopercepción & .0 & 12.0 & 2.13 & 2.31 & 63.72 & 63.62 & .98 \\
\hline & Memoria icónica & .0 & 9.0 & 5.94 & 1.90 & 86.20 & 67.05 & $.00^{* *}$ \\
\hline & Ritmo & .0 & 4.0 & 1.33 & 1.14 & 64.11 & 65.55 & .762 \\
\hline Edad & Prueba & Min & Max & M & $\mathrm{DE}$ & $\begin{array}{c}\text { M Centil } \\
\text { original }\end{array}$ & $\begin{array}{l}\text { M Centil } \\
\text { Colombia }\end{array}$ & Sig+ \\
\hline \multirow{7}{*}{$\begin{array}{c}4 \\
\text { años }\end{array}$} & Psicomotricidad & 5.0 & 11.0 & 7.79 & 1.27 & 61.37 & 61.88 & .90 \\
\hline & Lenguaje articulatorio & .0 & 15.0 & 10.63 & 3.58 & $57 ., 83$ & 56.59 & .79 \\
\hline & Lenguaje comprensivo & .0 & 9.0 & 3.83 & 2.01 & 47.03 & 46.70 & .94 \\
\hline & Estructuración espacial & 2.0 & 14.0 & 8.50 & 2.92 & 63.48 & 47.88 & $.01^{* *}$ \\
\hline & Visopercepción & 1.0 & 12.0 & 7.16 & 2.82 & 65.92 & 60.50 & .25 \\
\hline & Memoria icónica & 4.0 & 9.0 & 6.81 & 1.26 & 72.88 & 67.18 & .19 \\
\hline & Ritmo & .0 & 5.0 & 1.57 & 1.39 & 45.27 & 65.59 & $.00^{* *}$ \\
\hline Edad & Prueba & Min & Max & M & $\mathrm{DE}$ & $\begin{array}{c}\text { M Centil } \\
\text { original }\end{array}$ & $\begin{array}{l}\text { M Centil } \\
\text { Colombia }\end{array}$ & $p$ \\
\hline \multirow{7}{*}{$\begin{array}{c}5 \\
\text { años }\end{array}$} & Psicomotricidad & 4.0 & 11.0 & 8.31 & 1.53 & 55.79 & 60.92 & 34 \\
\hline & Lenguaje articulatorio & 1.0 & 15.0 & 11.79 & 3.62 & 58.83 & 59.16 & .95 \\
\hline & Lenguaje comprensivo & .0 & 9.0 & 4.37 & 2.20 & 47.64 & 41.48 & .27 \\
\hline & Estructuración espacial & 4.0 & 14.0 & 10.44 & 2.87 & 69.61 & 50.68 & $.00^{* *}$ \\
\hline & Visopercepción & 3.0 & 15.0 & 9.87 & 3.00 & 55.79 & 46.70 & .11 \\
\hline & Memoria icónica & 4.0 & 10.0 & 7.29 & 1.34 & 72.20 & 52.07 & $.00^{* *}$ \\
\hline & Ritmo & .0 & 9.0 & 2.20 & 1.85 & 32.66 & 53.75 & $.00^{* *}$ \\
\hline
\end{tabular}




\begin{tabular}{clccccccc}
\hline Edad & Prueba & Min & Max & M & DE & $\begin{array}{c}\text { M Centil } \\
\text { original }\end{array}$ & $\begin{array}{c}\text { M Centil } \\
\text { Colombia }\end{array}$ & $p$ \\
\hline \multicolumn{1}{c}{6} & Psicomotricidad & 5.0 & 11.0 & 8.92 & 1.36 & 61.63 & 69.13 & .22 \\
años & Lenguaje articulatorio & 1.0 & 15.0 & 12.47 & 3.94 & 59.07 & 66.42 & .32 \\
& Lenguaje comprensivo & .0 & 9.0 & 5.36 & 2.45 & 58.31 & 57.65 & .93 \\
& Estructuración espacial & 5.0 & 15.0 & 11.42 & 2.48 & 76.76 & 63.18 & $.04^{*}$ \\
& Visopercepción & 4.0 & 24.0 & 11.78 & 4.28 & 59.07 & 62.73 & .65 \\
& Memoria icónica & 5.0 & 10.0 & 7.92 & 1.36 & 74.31 & 61.92 & $.03^{*}$ \\
\cline { 2 - 8 } & Ritmo & .0 & 6.0 & 3.47 & 1.60 & 50.97 & 67.36 & $.01^{*}$ \\
\hline
\end{tabular}

Nota: Tabla autoría propia; ${ }^{*} p<.05 y{ }^{* *} p<.01$

+ Estadístico t-student para muestras independientes

Se presentan una aproximación al desempeño en la prueba del CUMANES, según sus valores interpretativos en el Índice de Desarrollo Neuropsicológico.

\section{Tabla 4}

Desempeño por Frecuencias en las subescalas con los baremos originales y baremos colombianos del CUMANES

\begin{tabular}{|c|c|c|c|c|c|c|c|c|c|c|c|c|c|c|}
\hline \multicolumn{15}{|c|}{ Desempeńo } \\
\hline & \multicolumn{2}{|c|}{$\begin{array}{c}\text { Muy } \\
\text { bajo n } \\
(\%)\end{array}$} & \multicolumn{2}{|c|}{$\begin{array}{l}\text { Bajo } \\
\mathrm{n}(\%)\end{array}$} & \multicolumn{2}{|c|}{$\begin{array}{c}\text { Medio bajo } \\
\text { n (\%) }\end{array}$} & \multicolumn{2}{|c|}{$\begin{array}{l}\text { Medio } \\
\text { n (\%) }\end{array}$} & \multicolumn{2}{|c|}{$\begin{array}{c}\text { Medio alto } \\
\mathrm{n}(\%)\end{array}$} & \multicolumn{2}{|c|}{$\begin{array}{l}\text { Alto } \\
\mathrm{n}(\%)\end{array}$} & \multicolumn{2}{|c|}{$\begin{array}{l}\text { Muy alto } \\
\text { n (\%) }\end{array}$} \\
\hline & $\mathrm{O}$ & B & $\mathrm{O}$ & B & $\mathrm{O}$ & B & $\mathrm{O}$ & B & $\mathrm{O}$ & B & $\mathrm{O}$ & B & $\mathrm{O}$ & B \\
\hline $\begin{array}{l}\text { Índice } \\
\text { de De- } \\
\text { sarrollo } \\
\text { Neurop- } \\
\text { sicoló- } \\
\text { gico }\end{array}$ & $\begin{array}{c}7 \\
(3.5)\end{array}$ & $\begin{array}{c}2 \\
(1)\end{array}$ & $\begin{array}{c}40 \\
(20)\end{array}$ & $\begin{array}{c}21 \\
(10.5)\end{array}$ & $\begin{array}{c}33 \\
(16.5)\end{array}$ & $\begin{array}{c}15 \\
(7.5)\end{array}$ & $\begin{array}{c}85 \\
(42.5)\end{array}$ & $\begin{array}{c}77 \\
(38.5)\end{array}$ & $\begin{array}{c}7 \\
(3.5)\end{array}$ & $\begin{array}{c}19 \\
(9.5)\end{array}$ & $\begin{array}{c}19 \\
(9.5)\end{array}$ & $\begin{array}{c}43 \\
(21.5)\end{array}$ & $\begin{array}{c}9 \\
(4.5)\end{array}$ & $\begin{array}{c}23 \\
(11.5)\end{array}$ \\
\hline
\end{tabular}

Tabla autoría propia: O: Baremo original español. B: Baremo colombiano 
Tomando como referencia los baremos originales en un $40 \%$ se encontraron puntuaciones muy bajas, bajas y media bajas, no obstante, tomando como referencia los baremos colombianos solo el 19\% se encuentra entre estos rangos de desempeño.

Se encontró que el $17.5 \%$ de los participantes tienen un desempeńo medio alto, alto y muy alto con los baremos originales. Mientras que, con el uso de baremos colombianos el $42.5 \%$ de los participantes tienen estas puntuaciones.

\section{Tabla 5}

Estadísticos descriptivos de las escalas baremadas del CUMANES

\begin{tabular}{|c|c|c|c|c|c|c|}
\hline Edad & Puntuación & Min & Max & M & $\mathrm{DE}$ & $p$ \\
\hline \multirow[t]{4}{*}{$\begin{array}{c}7 \\
\text { años }\end{array}$} & $\begin{array}{l}\text { Índice de Desarrollo Neuropsicológico } \\
\text { (original) }\end{array}$ & 74 & 139 & 103.82 & 16.37 & .109 \\
\hline & $\begin{array}{l}\text { Índice de Desarrollo Neuropsicológico } \\
\text { (colombiano) }\end{array}$ & 74 & 124 & 109.82 & 16.69 & \\
\hline & Percentil (original) & 4 & 99 & 55.75 & 30.25 & .011 \\
\hline & Percentil (colombiano) & 4 & 99 & 67.85 & 35.37 & \\
\hline Edad & Puntuación & Min & Max & M & $\mathrm{DE}$ & $p$ \\
\hline \multirow[t]{4}{*}{$\begin{array}{c}8 \\
\text { años }\end{array}$} & $\begin{array}{l}\text { Índice de Desarrollo Neuropsicológico } \\
\text { (original) }\end{array}$ & 74 & 139 & 105.10 & 18.23 & $.00^{* *}$ \\
\hline & $\begin{array}{l}\text { Índice de Desarrollo Neuropsicológico } \\
\text { (colombiano) }\end{array}$ & 76 & 139 & 121.18 & 21.61 & \\
\hline & Percentil (original) & 4 & 99 & 56.30 & 31.77 & $.00^{* *}$ \\
\hline & Percentil (colombiano) & 3,30 & 99 & 76.15 & 30.58 & \\
\hline Edad & Puntuación & Min & Max & $\mathrm{M}$ & $\mathrm{DE}$ & $p$ \\
\hline \multirow[t]{4}{*}{$\begin{array}{c}9 \\
\text { años }\end{array}$} & $\begin{array}{l}\text { Índice de Desarrollo Neuropsicológico } \\
\text { (original) }\end{array}$ & 76 & 124 & 95.82 & 11.09 & $.00^{* *}$ \\
\hline & $\begin{array}{l}\text { Índice de Desarrollo Neuropsicológico } \\
\text { (colombiano) }\end{array}$ & 76 & 124 & 107.62 & 16.51 & \\
\hline & Percentil (original) & 5 & 95 & 40.30 & 24.45 & $.00^{* *}$ \\
\hline & Percentil (colombiano) & 1,40 & 99 & 68.60 & 34.42 & \\
\hline
\end{tabular}




\begin{tabular}{|c|c|c|c|c|c|c|}
\hline Edad & Puntuación & Min & Max & M & $\mathrm{DE}$ & $p$ \\
\hline \multirow[t]{4}{*}{$\begin{array}{c}10 \\
\text { años }\end{array}$} & $\begin{array}{l}\text { Índice de Desarrollo Neuropsicológico } \\
\text { (original) }\end{array}$ & 68 & 118 & 83.17 & 12.23 & $.00^{* *}$ \\
\hline & $\begin{array}{l}\text { Índice de Desarrollo Neuropsicológico } \\
\text { (colombiano) }\end{array}$ & 69 & 106 & 96.02 & 12.59 & \\
\hline & Percentil (original) & 2 & 88 & 18.60 & 21.34 & $.00^{* *}$ \\
\hline & Percentil (colombiano) & 17,1 & 99 & 82.99 & 22.49 & \\
\hline \multirow[t]{4}{*}{$\begin{array}{c}11 \\
\text { años }\end{array}$} & $\begin{array}{l}\text { Índice de Desarrollo Neuropsicológico } \\
\text { (original) }\end{array}$ & 61 & 128 & 92.05 & 13.69 & $.00^{* *}$ \\
\hline & $\begin{array}{l}\text { Índice de Desarrollo Neuropsicológico } \\
\text { (colombiano) }\end{array}$ & 61 & 111 & 100 & 13.25 & \\
\hline & Percentil (original) & 1 & 97 & 34.40 & 25.80 & $.00^{* *}$ \\
\hline & Percentil (colombiano) & 1,30 & 99 & 77.37 & 28.69 & \\
\hline
\end{tabular}

Nota: Tabla autoría propia; ${ }^{*} p<.05 y{ }^{* *} p<.01$

+ Estadístico t-student para muestras independientes

Se encuentra cambios a nivel descriptivo en el uso de los datos normativos, evidenciando puntuaciones más altas en el uso de los baremos colombianos, esto se da en la media del IDN. Según valores interpretativos de los autores, los participantes de los grupos de edad de 7, 9, 10 y 11 años tienen un desempeño promedio, mientras que el grupo de 8 años tiene un desempeño alto. En cuanto al uso de los baremos españoles todos los grupos de edad poseen un desempeño medio, exceptuando el grupo de 10 años quienes presentan un desempeño bajo. A su vez se encuentran diferencias significativas en la calificación del IDN y los percentiles evaluados con los dos datos normativos, entre los grupos de edad de 8, 9, 10 y 11 años.

Se realiza el análisis del desempeño por cada una de las pruebas haciendo uso de los baremos colombianos elaborados en cada uno de los cuestionarios utilizados. 
Comparación de los baremos del CUMANIN y CUMANES / Chinome Torres y Rodríguez

\section{Tabla 6}

Diferencias según sexo en el desempeño de algunas subescalas del CUMANIN

\begin{tabular}{lcccc}
\hline Escala & Sexo & M & DE & $p$ \\
\hline Psicomotricidad & Femenino & 66.45 & 24.48 & .06 \\
& Masculino & 59.43 & 59.43 & \\
Lenguaje articulatorio & Femenino & 64.17 & 28.98 & $.03^{*}$ \\
& Masculino & 55.31 & 31.07 & \\
Lenguaje comprensivo & Femenino & 52.16 & 31.51 & .93 \\
& Masculino & 51.82 & 29.60 & \\
Estructuración espacial & Femenino & 54.25 & 30.32 & .60 \\
& Masculino & 56.46 & 30.55 & \\
Viso percepción & Femenino & 59.09 & 31.61 & .47 \\
& Masculino & 56.96 & 26.62 & \\
Memo icónica & Femenino & 63.47 & 27.90 & .47 \\
& Masculino & 60.63 & 27.60 & \\
Ritmo & Femenino & 61.51 & 24.24 & .48 \\
\hline
\end{tabular}

Nota: ${ }^{*} p \leq .05$

+ prueba t-student para muestras independientes

Únicamente se encuentra diferencias en la escala de lenguaje articulatorio, siendo superior las participantes de sexo femenino. 


\section{Tabla 7}

Diferencias según tipo de institución en el desempeño de algunas subescalas del CUMANIN

\begin{tabular}{llclc}
\hline Escala & Tipo & M & DE & $p$ \\
\hline Psicomotricidad & Privado & 60.69 & 26.68 & .18 \\
& Público & 65.82 & 26.20 & \\
Lenguaje articulatorio & Privado & 55.94 & 31.40 & $.03^{*}$ \\
& Público & 64.98 & 27.79 & \\
Lenguaje comprensivo & Privado & 49.30 & 32.36 & .15 \\
& Público & 55.70 & 27.64 & \\
Estructuración espacial & Privado & 52.49 & 30.23 & .10 \\
& Público & 59.79 & 30.61 & \\
Viso percepción & Privado & 58.15 & 30.54 & .86 \\
& Público & 58.91 & 27.65 & \\
Memo icónica & Privado & 64.23 & 26.76 & .20 \\
& Público & 59.16 & 28.87 & \\
Ritmo & Privado & 62.69 & 24.05 & .86 \\
\hline
\end{tabular}

Nota: ${ }^{*} p \leq .05$

+ prueba t-student para muestras independientes

Se encontraron diferencias únicamente en la escala de lenguaje articulatorio entre el tipo de institución, a nivel descriptivo se observa que el colegio público tuvo una mejor puntuación.

\section{Tabla 8}

Diferencias según sexo en el desempeño de algunas subescalas del CUMANES

\begin{tabular}{lcccc}
\hline Escala & Sexo & $\mathrm{M}$ & $\mathrm{DE}$ & $p$ \\
\hline Índice de Desarrollo & Femenino & 107.50 & 18.46 & .73 \\
Neuropsicológico & Masculino & 107.39 & 18.55 &
\end{tabular}


No se encontraron diferencias significativas según la variable sexo.

\section{Tabla 9}

Diferencias según el tipo de institución en el desempeño de algunas subescalas del CUMANES

\begin{tabular}{lcccc}
\hline Escala & Tipo de Institución & $\mathrm{M}$ & $\mathrm{DE}$ & $p$ \\
\hline Índice de Desarrollo & Privado & 112.15 & 18.14 & $.00^{* *}$ \\
Neuropsicológico & Público & 101.74 & 17.36 & \\
\hline
\end{tabular}

Nota: ${ }^{*} p \leq .05^{* *} p \leq .01$.

Se encontraron diferencias significativas en el IDN de la prueba CUMANES entre las instituciones de tipo público y privado, a nivel descriptivo se evidencia un mejor desempeño en los participantes pertenecientes a la institución privada, el valor medio corresponde a un desempeño medio alto, mientras que los pertenecientes a la institución pública tienen un desempeño promedio.

\section{Discusión}

El objetivo de la investigación fue realizar una evaluación neuropsicológica aplicando los baremos colombianos de los Cuestionarios de Madurez Neuropsicológica Infantil (CUMANIN) y Escolar (CUMANES) en una muestra de nińos con edades comprendidas entre los 3 y 11 años. A su vez realizar una comparación entre los baremos originales españoles y baremos colombianos y describir las posibles diferencias.

Con respecto a la valoración con el CUMANIN, a partir de los hallazgos en las tablas 2 y 3 , se encontraron diferencias en el desempeño por subescala de acuerdo al baremo a utilizar, en general el uso del baremo espańol, mostró menos frecuencias en el desempeño bajo y mayores frecuencias en el desempeño medio y alto, lo cual daría algunas ventajas en términos de desempeño frente al baremo colombiano y su 
aproximación diagnóstica a su vez variaría, por otro lado, se encontraron diferencias en algunas subescalas de acuerdo al grupo de edad a partir del análisis estadístico t-student. En general se encontró que existe un desempeño en aumento, conforme la edad de los niños avanza, de manera similar como se ha encontrado en otras investigaciones (Chinome Torres et al., 2017; Parra-Pulido et al., 2016; Urzúa et al., 2010). Esto puede ser explicado por el desarrollo sináptico progresivo que sucede en estas etapas del desarrollo y que van a ser determinantes a lo largo del ciclo vital (Gilman et al., 2017; Knickmeyer et al., 2008; Walhovd et al., 2016)but identification of how early factors impact human brain and cognition throughout life has remained challenging. Much focus has been on age-specific mechanisms affecting neural foundations of cognition and their change. In contrast to this approach, we tested whether cerebral correlates of general cognitive ability (GCA.

Se encontró que haciendo uso del baremo colombiano cerca del $21.2 \%$ de los participantes en promedio había obtenido un desempeño bajo y el 33.3\% obtuvieron puntuaciones medias en alguna de las subescalas del CUMANIN, dando un aproximado al nivel madurativo de los participantes, en cuanto al CUMANES (tabla 4) con el baremo colombiano se encontró que el 19\% de los participante obtuvieron puntuaciones muy bajas, bajas y media bajas. De manera similar que en la presente investigación, se ha encontrado investigaciones donde se detectan niveles de inmadurez en un 53\% de la muestra (Chinome Torres et al., 2017), otra investigación refiere un bajo desempeño en un $19.7 \%$ y en un $22.9 \%$ un nivel esperado (Ternera, Molina y Ternera, 2012) en el estudio de Guamani Toapanta (2016) se encontró que el $43 \%$ de su muestra no obtuvieron puntuaciones deseables asociadas al desarrollo madurativo normal, en la investigación de Parra-Pulido et al. (2016) se encontró que el 63\% de su muestra obtuvieron puntuaciones por debajo de lo esperado en 2 o más subescalas del CUMANIN.

A través del análisis comparativo entre el tipo de institución y la variable sexo, se encontraron diferencias significativas únicamente en la escala de lenguaje articulatorio (tablas 6 y 7). Respecto al desempeńo en lenguaje articulatorio superior en las mujeres se ha encontrado que 
la estructura cerebral es diferente entre sexos, su actividad cortical y densidad es diferente, por tanto su estructura mental también lo es (Alonso-Nanclares et al.,2008). En el estudio de Ingalhalikar et al. (2014) se encontró que los hombres tenían mejores conexiones a nivel intrahemisférico situación que mejoraba su desempeño en percepción y acción coordinada, mientras que las mujeres poseían mejores conexiones a nivel interhemisférico aspecto que mejoraba su procesamiento analítico e intuitivo. Eespecíficamente en lenguaje, se encontró un mejor desempeño en un estudio realizado en niños de Chile y Nicaragua, donde las niñas tuvieron un mejor desempeño en esta área y en las habilidades socioemocionales (Bando, López-Boo y Li, 2016).

Se encontró a nivel descriptivo un mejor desempeño en lenguaje articulatorio en las instituciones públicas, aunque no es lo esperado, la bibliografía científica ha encontrado mejores puntuaciones en el desempeño en las instituciones privadas, situación denominada brechas en el desempeño escolar, y se atribuye sobre todo a mejores condiciones socioeconómicas y escolaridad de los padres(Castro et al., 2016), el lenguaje articulatorio depende en parte de la socialización del individuo con su entorno (Portellano Pérez et al., 2000), puede ser posible que por un mayor número de estudiantes escolarizados en los colegios públicos tengan mayor oportunidad para socializar con sus iguales, aunque es una aseveración que requiere investigación a mayor profundidad.

En cuanto a los hallazgos con el CUMANES, se encontraron diferencias con respecto al uso de los baremos por edad en el IDN y los percentiles (tabla 5), se encuentran mayores puntuaciones haciendo uso de los baremos colombianos, situación que modificaría significativamente la interpretación y aproximación diagnóstica en el desempeño, esto se corrobora en la tabla 4 de acuerdo con los porcentajes obtenidos en cada uno de los valores interpretativos. No se hallaron diferencias significativas en el desempeño de acuerdo al sexo (tabla 8), se encontraron diferencias de acuerdo al tipo de institución, a nivel descriptivo se observa un mejor desempeño en el colegio privado (tabla 9) tal como se hacía mención obedece a brechas educativas lo cual tiene relación con las experiencias tempranas, el desarrollo cerebral óptimo en 
ambientes enriquecidos y factores socioeconómicos familiares y contextuales (Cermakova et al., 2018; Nelson et al., 2007, 2009; Smyke et al., 2007; Waber et al., 2018; Walker et al., 2011b).

En conclusión, para la evaluación psicológica y neuropsicológica se desarrollan baterías válidas y fiables, cuyo propósito es de manera detallada analizar un constructo en particular, por lo general sus estándares se desarrollan en población anglosajona, situación que dificulta su uso e interpretación (González-Pérez et al., 2014) esto representa una debilidad pues no se cuentan con estándares propios que permitan una comparación con un grupo normativo poblacional.

Para Abad et al. (2016) un baremo es un valor numérico que se asigna a un individuo y a partir de este valor comparar su desempeño con un grupo normativo de tal forma que se cumple con la comparación de edad y otras variables compartidas que permiten reducir diversos sesgos. A pesar de las diferencias halladas entre los baremos utilizados en los cuestionarios, es importante que investigadores y profesionales sigan las recomendaciones concernientes al uso de pruebas psicométricas en la valoración psicológica que dicta la American Psychological Association (APA), y que diversos entes gubernamentales han añadido en sus códigos éticos y deontológicos que regulan la profesión en psicología a nivel mundial.

Entre las sugerencias se destaca el uso apropiado de test, la construcción de test, la interpretación de los resultados en las valoraciones, el uso apropiado de las evaluaciones y los servicios de interpretación y calificación de las evaluaciones psicológicas, dichos componentes en mención sugieren a los profesionales en psicología contar con test que tengan las herramientas psicométricas necesarias para dar una aproximación diagnóstica adecuada, tener certeza de la evidencia científica que respalda el test a utilizar, conocer la fiabilidad, validez y estudios sobre la estandarización y resultados de los test, identificar las situaciones en las cuales técnicas de evaluación, o normativas pueden no ser aplicables, o pueden requerir un ajuste en la aplicación o interpretación, debido a características de la población (American Psychological Association, 1992, 2010; Messick, 1980). 
Por lo anterior se sugiere hacer uso de los baremos que tengan compatibilidad con la población a quien va dirigida la valoración psicológica, ya que esto supone contar con un valor de referencia, sin embargo, es necesario acatar las sugerencias previamente descritas y cumpliendo con lo sugerido por el comité de ética de la APA, además, de contar con diversas fuentes de recolección de información, esto con el fin de realizar una valoración integral y contar con más herramientas de valoración psicológica que permitan dar una aproximación diagnóstica con mayor validez.

El uso de pruebas psicométricas supone una ayuda importante en el momento de realizar aproximaciones diagnósticas de acuerdo con el problema a analizar, en algunos países el uso de estas pruebas es escaso y en su mayoría no se cuentan con valores de referencia para su población especifica. Lo anterior supone un reto, pues en la elaboración de políticas gubernamentales es necesario contar con un diagnóstico válido para orientar estrategias que logren la mitigación de diversas problemáticas, por esta razón contar con herramientas científicas válidas disminuiría el sesgo en la interpretación de las pruebas.

\section{Referencias}

Abad, F., Garrido, J., Olea, J. y Ponsoda, V. (2016). Introducción a la psicometría: teoría clásica de los test y teoría de la respuesta al item. Universidad Autónoma de Madrid.

Ackerman, B. P. y Brown, E. D. (2006). Income poverty, poverty cofactors, and the adjustment of children in elementary school. Advances in Child Development and Behavior, 34, 91-129. http:// doi.org/10.1016/S0065-2407(06)80005-4

Alonso-Nanclares, L., Gonzalez-Soriano, J., Rodriguez, J. R. y DeFelipe, J. (2008). Gender differences in human cortical synaptic density. Proceedings of the National Academy of Sciences of the United States of America, 105(38), 14615-14619. http://doi. org/10.1073/pnas.0803652105 
American Psychological Association, E. C. (1992). Ethical Principles of Psychologists and Code of Conduct. American Psychologist, 47(12), 1597-1611. http://doi.org/10.1037/0003066X.47.12.1597

American Psychological Association (2010). 2010 Amendments to the 2002 "Ethical principles of psychologists and code of conduct". American Psychologist. American Psychological Association. http://doi.org/10.1037/a0020168

Arango-Lasprilla, J., Rivera, D (2015). Neuropsicología en Colombia: Datos normativos, estado actual y retos a futuro. Editorial Autónoma de Manizales

Ávila, A. M. (2012). Adaptacion del cuestionario de madurez Neuropsicológica Infantil Cumanin de Portellano. Revista Iberoamericana de Psicología: Ciencia y Tecnología, 5(1), 91-99.

Bando, R., López-Boo, F. y Li, X. (2016). Sex-differences in language and socio-emotional skills in early childhood. Office of Strategic Planning and Development Effectiveness Social Protection and Health Division Inter-American Development Bank. Recuperado de http://www.iadb.org

Benítez, Y. R., Bringas, M. D., Jiménez-Morales, R. M. y Macías, V. E. F. (2015). Normas cubanas del instrumento neuropsicológico Luria Inicial para nińos preescolares 4-6 años. Cuadernos de Neuropsicologia Panamerican Journal of Neuropshychology, 9(2), 49-70. Recuperado de http://10.0.30.34/cnps/9.2.203

Black, M. M., Walker, S. P., Fernald, L. C. H., Andersen, C. T., DiGirolamo, A. M., Lu, C., McCoy, D.,Fink, G., Shawar, Y., Shiffman, J., Devercelli, A., Wondon, Q., Vargas-Barón, E. \& GranthamMcGregor, S. (2016). Early childhood development coming of age: science through the life course. The Lancet, 380(10064), 1-14. http://doi.org/10.1016/S0140-6736(16)31389-7

Bryck, R. L. y Fisher, P. A. (2012). Training the brain: Practical applications of neural plasticity from the intersection of cognitive neuroscience, developmental psychology, and prevention 
science. American Psychologist, 67(2), 87-100. http://doi. org/10.1037/a0024657

Castro, G., Díaz, A. M., Jairo, R. y Bedoya, T. (2016). Documentos de Trabajo Fcea Departamento de Economía, 26. Recuperado de https://www.javerianacali.edu.co/sites/ujc/files/node/fielddocuments/field_document_file/ddtn262016.pdf

Cermakova, P., Formanek, T., Kagstrom, A. y Winkler, P. (2018). Socioeconomic position in childhood and cognitive aging in Europe. Neurology, 91(17), e1602-e1610. http://doi. org/10.1212/WNL.0000000000006390

Chinome Torres, J. D., Barreto, R., Carlota, L. y Parra Pulido, J. H. (2017). Implementación y evaluación de un programa de estimulación cognitiva en preescolares rurales. Psicología desde el Caribe, 34(3), 184-203.

Colmenero, F. B. y Robles Bello, M. A. (2012). Utilidad del cuestionario CUMANIN para detectar diferencias, en dos grupos de preescolares, dentro de un programa de Atención Temprana. (Spanish). Utility of the CUMANIN Questionnaire for Detecting Differences in Two Groups of Preschool Aged Children in an Early Care Program. (English). Electronic Journal of Research in Educational Psychology, 10(1) 311-332.

Fritze, T., Doblhammer, G. y van den Berg, G. J. (2014). Can individual conditions during childhood mediate or moderate the long-term cognitive effects of poor economic environments at birth? Social Science and Medicine, 119(2014) 240-248. http:// doi.org/10.1016/j.socscimed.2014.07.011

Gilman, S. E., Hornig, M., Ghassabian, A., Hahn, J., Cherkerzian, S., Albert, P. S., Buka, L. y Goldstein, J. M. (2017). Socioeconomic disadvantage, gestational immune activity, and neurodevelopment in early childhood. Proceedings of the National Academy of Sciences of the United States of America, 114(26), 6728-6733. http://doi.org/10.1073/pnas.1617698114

González-Pérez, P. A., Hernández, S., Martín-González, R., Verche, E., Quintero, I., Bravo, J. y García-Marco, E. (2014). Memoria 
Visual (Dcs): Neurodesarrollo de la Memoria Figurativa [Adaptation in Children Population of the Visual Learning and Memory Test for Neuropsychological Assessment (DCS): Neurodevelopment of the Figurative Memory]. Acción Psicológica, 10(2), 115-126. http://doi.org/10.5944/ap.10.2.12215

Guamani Toapanta, G. M. (2016). La estimulación kinestésica y su influencia en el desarrollo cognitivo en niños de 4 a 5 años del Centro Educativo Nueva Era. Recuperado de http://repo.uta. edu.ec/handle/123456789/23165

Guerrero-leiva, M. K. (2006). Adaptación del Cuestionario de Madurez Neuropsicológica Infantil-CUMANIN en una población urbana de Lima. Herediana 1(1), 66-75 Recuperado de http://biblio.uarm.edu.pe/cgi-bin/koha/opac-detail. pl?biblionumber $=61542 \% 5 \mathrm{Cnhttps}$ ://www.researchgate.net/ publication/237356921_Adaptacion_del_Cuestionario_de_ Madurez_Neuropsicologica_Infantil-CUMANIN_en_una_ Poblacion_Urbana_de_Lima

Heckman, J. J. y Masterov, D. V. (2007). The productivity argument for investing in young children. Review of AgriculturalEconomics, (29), 446-493. http://doi.org/10.1111/j.1467-9353.2007.00359.x

Hernandez Sampieri, R., Fernandez Collado, C. y Baptista Lucio, M. del P. (2010). Metodología de la investigación (10th ed.). McGrawHill. http://doi.org/-ISBN 978-92-75-32913-9

Ingalhalikar, M., Smith, A., Parker, D., Satterthwaite, T. D., Elliott, M. A., Ruparel, K., Hakonarson, H., Gur, R., Gur, R. y Verma, R. (2014). Sex differences in the structural connectome of the human brain. Proceedings of the National Academy of Sciences of the United States of America, 111(2), 823-828. http://doi. org/10.1073/pnas.1316909110

John, C. C., Black, M. M. y Nelson, C. A. (2017). Neurodevelopment: The impact of nutrition and inflammation during early to middle childhood in low-resource settings. Pediatrics. http:// doi.org/10.1542/peds.2016-2828H 
Johnson, S. B., Riis, J. L. y Noble, K. G. (2016). State of the art review: poverty and the developing brain. Pediatrics, 137(4) 1-16. http:// doi.org/10.1542/peds.2015-3075

Knickmeyer, R. C., Gouttard, S., Kang, C., Evans, D., Wilber, K., Smith, J. K., Hamer, RM., Lin, W., Gerig, G. y Gilmore, J. H. (2008). A structural MRI study of human brain development from birth to 2 years. J Neurosci, 28(47), 12176-12182. http:// doi.org/10.1523/JNEUROSCI.3479-08.2008\r28/47/12176 [pii]

Lenroot, R. K. y Giedd, J. N. (2006). Brain development in children and adolescents: Insights from anatomical magnetic resonance imaging. Neuroscience and Biobehavioral Reviews, 30(6) 18-29. http://doi.org/10.1016/j.neubiorev.2006.06.001

Martín-Casas, P., Ballestero-Pérez, R., Meneses-Monroy, A., BeneitMontesinos, J. V., Atínn-Arratibel, M. A. y Portellano-Pérez, J. A. (2015). Neurodevelopment in preschool idiopathic toewalkers. Neurologia, 32(7) 446-454. http://doi.org/10.1016/j. nrl.2016.02.012

Martín Casari, L. y Oros Cabrini, M. P. (2013). Una experiencia de evaluación psicológica en una zona rural. PSIENCIA: Revista Latinoamericana de Ciencia Psicológica 5(2), 150-158. Recuperado de https://www.redalyc.org/pdf/3331/333129928014.pdf

Martin-Casas, P., Meneses-Monroy A., Atín-Arratibel, M. y BeneitMontesinos, J. V. (2016). Decision trees: linking biomechanical and developmental characteristics of preschool idiopathic toe walkers: A cross-sectional study, Austin Pediatrycs 3(3), 1036. Recuperado de https:/austinpublishinggroup.com/pediatrics/ fulltext/ajp-v3-id1036.php

McDermott, C. L., Seidlitz, J., Nadig, A., Liu, S., Clasen, L. S., Blumenthal, J. D., Raznahan, A. (2018). Longitudinally mapping childhood socioeconomic status associations with cortical and subcortical morphology. Journal of Neuroscience, 39(8) 1365-1373. http://doi.org/10.1101/352187 
Messick, S. (1980). Test validity and the ethics of assessment. American Psychologist, 35(11) 1012-1027. http://doi.org/10.1037/0003066X.35.11.1012

Millana-Cuevas., Portellano, M.-A. (2007). Alteraciones neuropsicológicas en niños infectados por el virus de inmunodeficiencia humana. Revue Neurologique, 44(6), 366-374. https://doi. org/10.33588/rn.4406.2005612

Moreno-Gómez, A.-J. y Cejudo, J. (2016). Effectiveness of a Mindfulness-Based Social-Emotional Learning Program on psychosocial adjustment and neuropsychological maturity in kindergarten children. Mindfulness, 10, 111-121. http://doi.org/10.1007/ s12671-018-0956-6

Nell, V. (2000). Cross-cultural neuropsychological assessment: Theory and practice. Mahwah, NJ: Lawrence Erlbaum Associates Publishers.

Nelson, C. A., Furtado, E. A., Fox, N. A. y Zeanah, C. H. (2009). The deprived human brain. American Scientist, 97(3), 222-229. http://doi.org/10.1511/2009.78.222

Nelson, C. A., Zeanah, C. H., Fox, N. A., Marshall, P. J., Smyke, A. T. y Guthrie, D. (2007). Cognitive recovery in socially deprived young children: the Bucharest Early Intervention Project. Science (New York, N.Y.), 318(5858), 1937-1940. http://doi. org/10.1126/science. 1143921

Noble, K. G., Houston, S. M., Brito, N. H., Bartsch, H., Kan, E., Kuperman, J. M., Akshoomoff , N., Amaral, D., Bloss, C., Libiger, O., Schork, N., Murray, S., Casey, B., Chang, L., Ernst, T., Frazier, J., Gruen, J., Kennedy, D., Van Zijl, P., ... Sowell, E. R. (2015). Family income, parental education and brain structure in children and adolescents. Nature Neuroscience, 18, 773-778. http://doi.org/10.1038/nn.3983

Parra-Pulido, J. H., Rodríguez-Barreto, L. C. y Chinome-Torres, J. D. (2016). Evaluación de la madurez neuropsicológica infantil en preescolares. Revista Universidady Salud, 18(1), 126-137. Recuperado de http://www.scielo.org.co/pdf/reus/v18n1/v18n1a13. pdf 
Parra Pulido, J. H., Rodríguez Barreto, L. C. y Chinome Torres, J. D. (2015). Relación entre peso al nacer y madurez neuropsicológica en preescolares de Tunja (Colombia). Pensamiento Psicológico, 13(2), 65-77. http://doi.org/10.11144/Javerianacali.PPSI13-2. rpnm

Peng, L.-N., Cheng, Y., Chen, L.-K., Tung, H.-H., Chu, K.-H. y Liang, S.-Y. (2015). Cognition and social-physiological factors associated with malnutrition in hospitalized older adults in Taiwan. Journal of Nursing Research, 23(1), 1-5. http://doi.org/10.1097/ jnr.0000000000000074

Pérez-Escamilla, R., Rizzoli Córdoba, A., Alonso Cuevas, A. y Reyes Morales, H. (2017). Advances in early childhood development: from neurons to large scale programs. Boletín Médico del Hospital Infantil de México (English Edition), 74(2), 86-97. http:// doi.org/10.1016/j.bmhime.2017.11.022

Portellano, J. A., Mateos, R. y Martínez-Arias, R. (2012). Cuestionario de Madurez Neuropsicológica Escolar (CUMANES). TEA Ediciones.

Portellano Pérez, J. A., Mateos Mateos, R., Martínez Arias, R., Tapia Pavón, A. y Granados García-Tenorio, M. J. (2000). Cuestionario de madurez neuropsicológica infantil: CUMANIN. TEA. Recuperado de https://dialnet.unirioja.es/servlet/libro?codigo=219925

Rees, P., Booth, R. y Jones, A. (2016). The emergence of neuroscientific evidence on brain plasticity: Implications for educational practice. Educational \& Child Psychology, 33(1), 8-19.

Richter, L. M., Daelmans, B., Lombardi, J., Heymann, J., Boo, F. L., Behrman, J. R., Lu, C., Lucas, J., Perez-Escamilla, R., Dua, T., Bhutta, Z., Stenberg, K., Gertler, D. y Darmstadt, G. L. (2017). Investing in the foundation of sustainable development: pathways to scale up for early childhood development. The Lancet, 389(10024), 103-118. http://doi.org/10.1016/ S0140-6736(16)31698-1

Savanur, M. S., Purandare, M. y Ghugre, P. S. (2015). Influence of birth weight and stunting on selected cognitive functions of 
young children in low socioeconomic areas of Mumbai City. Journal of Psychosocial Research.

Schoenmaker, C., Juffer, F., van IJzendoorn, M. H., van den Dries, L., Linting, M., van der Voort, A. y Bakermans-Kranenburg, M. J. (2015). Cognitive and health-related outcomes after exposure to early malnutrition: The Leiden longitudinal study of international adoptees. Children and Youth Services, 48, 80-86. http:// doi.org/10.1016/j.childyouth.2014.12.010

Shawar, Y. R. y Shiffman, J. (2017). Generation of global political priority for early childhood development: the challenges of framing and governance. The Lancet, 389(10064), 119-124. http://doi. org/10.1016/S0140-6736(16)31574-4

Shonkoff, J. P. (2011). Protecting Brains, Not Simply Stimulating Minds. Science, 333(6045), 982-983. http://doi.org/10.1126/ science. 1206014

Smyke, A. T., Koga, S. F., Johnson, D. E., Fox, N. A., Marshall, P. J., Nelson, C. A. y Zeanah, C. H. (2007). The caregiving context in institution-reared and family-reared infants and toddlers in Romania. Journal of Child Psychology and Psychiatry and Allied Disciplines, 48(2), 210-218. http://doi. org/10.1111/j.1469-7610.2006.01694.x

Solís-Rodríguez, A. A., Morales-Sadaña, A., González-Bocaranda, J., Champsaur-Gómez, D. y Rodríguez-Ashby, A. (2018). Utilidad de una evaluación neuropsicológica informatizada de la memoria verbal, el Cogval-Verbal, para la detección del deterioro cognitivo. Datos preliminares. Invest. Pens. Crit, 6(1), 5-16. https:// doi.org/10.37387/ipc.v6i1.77

Sun, X., McHale, S. M. y Updegraff, K. A. (2017). Maternal and paternal resources across childhood and adolescence as predictors of young adult achievement. Journal of Vocational Behavior, 100, 111-123. http://doi.org/10.1016/j.jvb.2017.03.003

Ternera, C. C., Molina, R. T. y Ternera, L. C. (2012). Relación entre el grado de madurez neuropsicológica infantil y el índice de talla y peso en nińos de 3 a 7 ańos escolarizados de estratos socioeconó- 
micos dos y tres de la ciudad de barranquilla (Colombia). Salud Uninorte, 28(1), 88-98. Recuperado de http://www.scielo.org. co/scielo.php?script=sci_arttext\&pid=S0120-55522012000100 009\&lng=en \&nrm=iso.

Urzúa, A., Ramos, M., Alday, C. y Alquinta, A. (2010). Madurez neuropsicológica en preescolares: Propiedades psicométricas del test CUMANIN. Terapia Psicologica, 28(1), 13-25. http://doi. org/10.4067/S0718-48082010000100002

Waber, D. P., Bryce, C. P., Girard, J. M., Fischer, L. K., Fitzmaurice, G. M. y Galler, J. R. (2018). Parental history of moderate to severe infantile malnutrition is associated with cognitive deficits in their adult offspring. Nutritional Neuroscience, 21(3), 195-201. http://doi.org/10.1080/1028415X.2016.1258379

Walhovd, K. B., Krogsrud, S. K., Amlien, I. K., Bartsch, H., Bjørnerud, A., Due-Tønnessen, P., Donald, G., Asta, H., Håberg, W., Kremen, L., Ferschman, L., Panizzon, M., Rohani, D., Storsve, A., Sølsnes, A., Tamnes, C., Thompson, W., Reuter, C., Dale, A. y Fjell, A. M. (2016). Neurodevelopmental origins of lifespan changes in brain and cognition. Proceedings of the National Academy of Sciences of the United States of America, 113(33), 9357-62. http://doi.org/10.1073/pnas.1524259113

Walker, S. P., Wachs, T. D., Grantham-Mcgregor, S., Black, M. M., Nelson, C. A., Huffman, S. L., ... Richter, L. (2011a). Inequality in early childhood: Risk and protective factors for early child development. The Lancet, 378(9799), 1325-1338. http://doi. org/10.1016/S0140-6736(11)60555-2

Walker, S. P., Wachs, T. D., Grantham-Mcgregor, S., Black, M. M., Nelson, C. A., Huffman, S. L., Baker-Henningham, H., Chang, S., Hamadani, J., Lozoff, B., Meeks, J., Powell, C., Rahman, A \& Richter, L. (2011b). Inequality in early childhood: Risk and protective factors for early child development. The Lancet, 378(9799), 1325-1338. http://doi.org/10.1016/ S0140-6736(11)60555-2 
Walker, S. P., Wachs, T. D., Meeks Gardner, J., Lozoff, B., Wasserman, G. A., Pollitt, E. y Carter, J. A. (2007). Child development: risk factors for adverse outcomes in developing countries. The Lancet, 369(9556), 145-157. http://doi.org/10.1016/ S0140-6736(07)60076-2

Zamora Crespo, B. y García-Navarro, C. (2014). Pruebas neuropsicológicas en pediatría. Anales de Pediatría Continuada, 12(04), 191-197. Recuperado de http://www.apcontinuada.com/es/ pruebas-neuropsicologicas-pediatria/articulo/90339622/

Recibido: 2018-12-03

Revisado: 2021-11-17

Aceptado: 2021-11-22 